\title{
An analysis of the vertical photospheric velocity field as observed by THEMIS ${ }^{\star}$
}

\author{
V. Carbone ${ }^{1,2}$, F. Lepreti ${ }^{1,2}$, L. Primavera ${ }^{1,2}$, E. Pietropaolo ${ }^{3}$, F. Berrilli ${ }^{4}$, \\ G. Consolini ${ }^{5}$, G. Alfonsi ${ }^{6}$, B. Bavassano ${ }^{5}$, R. Bruno ${ }^{5}$, A. Vecchio ${ }^{1}$, and P. Veltri ${ }^{1,2}$ \\ 1 Dipartimento di Fisica, Università della Calabria, 87036 Rende (CS), Italy \\ 2 Istituto Nazionale per la Fisica della Materia, Unità di Cosenza, Italy \\ 3 Dipartimento di Fisica, Univesità di L'Aquila, 67010 L'Aquila, Italy \\ 4 Dipartimento di Fisica, Università di Roma "Tor Vergata", 00133 Roma, Italy \\ ${ }^{5}$ Istituto di Fisica dello Spazio Interplanetario-CNR, 00133 Roma, Italy \\ ${ }^{6}$ Dipartimento di Difesa del Suolo, Università della Calabria, 87036 Rende (CS), Italy
}

Received 3 April 2001 / Accepted 10 April 2001

\begin{abstract}
We propose the application of Proper Orthogonal Decomposition (POD) analysis to the photospheric vertical velocity field obtained through the data acquired by the THEMIS telescope, to recover a proper optimal basis of functions. As first results we found that four modes, which are energetically dominant, are nearly sufficient to reconstruct both the convective field and the field of the "5-min" oscillations.
\end{abstract}

Key words. Sun: photosphere - Sun: granulation - Sun: oscillations

\section{Introduction}

Photospheric periodic motions, known as "5-min" oscillations, have been observed since 1962 (Leighton et al. 1962). These oscillations have a period of about 3-12 min and are usually called $p$-modes. Models of these oscillations can be made by assuming that they are driven by pressure perturbations which provide the restoring force (see for example Stix 1991, and references therein). Usually, the observed line of sight velocity field $u(x, y, t)$ is analysed, in both time and space (the coordinates $(x, y)$ correspond to the position on the field of view), through a Fourier transform

$u(x, y, t)=\int v\left(k_{x}, k_{y}, \omega\right) \mathrm{e}^{i\left(k_{x} x+k_{y} y+\omega t\right)} \mathrm{d} k_{x} \mathrm{~d} k_{y} \mathrm{~d} \omega$.

The average power $P(k, \omega)$ (where $k^{2}=k_{x}^{2}+k_{y}^{2}$ ), defined through the Fourier coefficients of the velocity field

$P(k, \omega)=\frac{1}{2 \pi} \int_{0}^{2 \pi}|v(k \cos \phi, k \sin \phi, \omega)|^{2} \mathrm{~d} \phi$

Send offprint requests to: V. Carbone,

e-mail: carbone@fis.unical.it

* Based on observations made with THEMIS-CNRS/INSU-

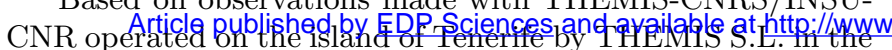
Spanish Observatorio del Teide of the Instituto de Astrofisica de Canarias. is not evenly distributed in the $k-\omega$ plane. Rather, it follows certain ridges, each corresponding to a certain fixed number of wave modes in the radial direction.

A different phenomenon usually observed on the same time scales on the photosphere is granulation, that is, a cellular pattern which covers the entire solar surface, except in sunspots. This pattern is commonly interpreted as the result of the turbulent convective motions which take place in the subphotospheric layers (see for example Bray et al. 1984). Since the time scales of $p$-mode oscillations and convective motions are of the same order, to measure convective velocities, the oscillatory contribution should be filtered out. This has become a standard technique by using Fourier transforms in both space and time (Title et al. 1989; Straus et al. 1992). The result is a map of the velocity field $u_{\mathrm{F}}(x, y, t)$ obtained by reconstructing the field through a filtered inverse Fourier transform. That is, the contribution of the ridges in the $k-\omega$ plane due to "5-min" oscillations is removed.

Fourier analysis has some disadvantages. The velocity field is represented as a linear combination of plane waves whose shape is given a priori, each corresponding to a characteristic scale $\left(\sim k^{-1}\right)$ and a characteristic pe-

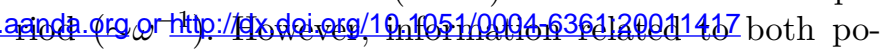
sition and time in physical space is completely hidden. This is an advantage when dealing with waves, but it is a 
strong disadvantage when dealing with strongly localised spatial structures like the convective structures. On the other hand, even a rough look at a typical granulation pattern of the solar photosphere (or a look at a high resolution map of ridge in the $k-\omega$ plane), yields the impression that most scales are excited (Consolini et al. 1999), and evolve stochastically in time. That is, the granulation is a complex spatio-temporal turbulent effect. We then need information about different scales, information that is often a useful ingredient for modelling and physical insight. This difficulty calls for a representation that decomposes the velocity field into contributions of different scales as well as different locations.

In the present paper we would like to introduce a different technique which allows us to decompose the velocity field, or other fields, thus providing a basis that optimally represents a flow in the energy norm. This is called Proper Orthonormal Decomposition (POD) and it is also known as the Karhunen-Loéve expansion. POD was introduced some time ago in the context of turbulence by Lumley (Lumley 1967; see also Holmes et al. 1998, and references therein), and it is a powerful technique to extract basis functions that represent ensemble averaged structures, such as coherent structures in turbulent flows. Convective structures, which are usually observed on the photosphere, can be seen as a kind of coherent structure within a stochastic field, and this represents the basis for the present paper. In the next section we briefly describe the POD, then we present the result of POD applied to velocity fields observed by THEMIS, and finally we discuss the results we obtained and future perspectives.

\section{Proper orthogonal decomposition}

POD is designed to yield a complete set of eigenfunctions that are optimal in energy, when compared to each other (Lumley 1967). Given an ensemble of fields, in our case the vertical velocity fields at different times $t$, namely $\{u(x, y, t)\}$, we introduce the expansion using the POD as

$u(x, y, t)=\sum_{j=0}^{\infty} a_{j}(t) \Psi_{j}(x, y)$

where the coefficients $a_{j}(t)$ are the temporal modal coefficients and $\Psi_{j}(x, y)$ are the POD eigenfunctions. We used a two-dimensional representation of the fields, with a single component, because this is the case at hand. The extension to the three-dimensional case with three components for the field is trivial from a theoretical point of view. The optimability of POD derives from the fact that a truncated POD describes typical members of the ensemble better than any other decomposition of the same truncation (Holmes et al. 1998). To construct the base we have to maximize the averaged projection of $u(x, y, t)$ onto $\Psi(x, y)$ constrained to the unitary norm $\|\Psi(x, y)\|=1$. In our paper we use the time averaging. This leads to a

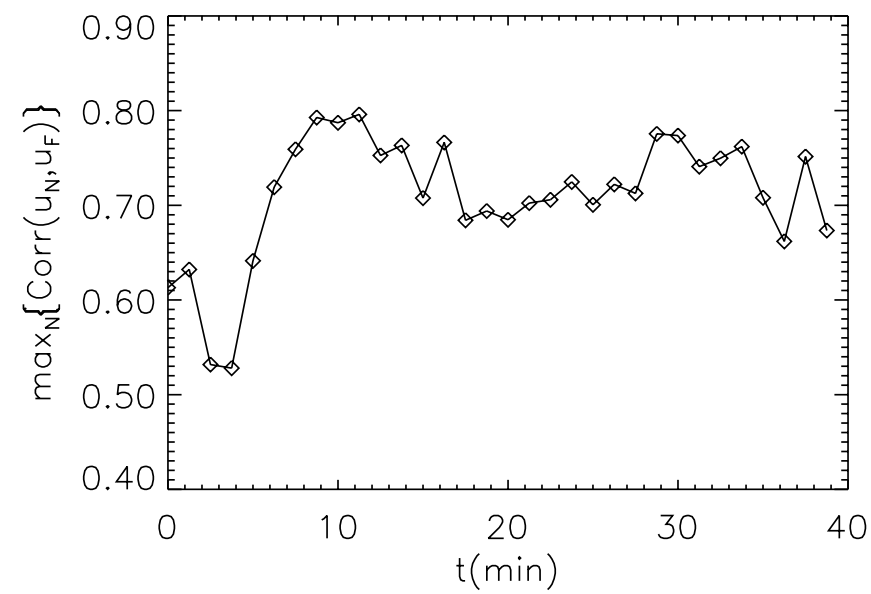

Fig. 1. We report the time evolution of the maximum crosscorrelation $\max C\left(u_{N}, u_{\mathrm{F}}\right)$ between the velocity field $u_{\mathrm{F}}(x, y, t)$, obtained through a filter by the $k-\omega$ technique, and the field $u_{N}(x, y, t)$ obtained through the POD procedure with a truncation to $N$ modes.

constrained optimization problem that can be cast as a Fredholm integral equation

$\int_{L_{x}} \int_{L_{y}} R\left(x, y ; x^{\prime}, y^{\prime}\right) \Psi\left(x^{\prime}, y^{\prime}\right) \mathrm{d} x^{\prime} \mathrm{d} y^{\prime}=\lambda \Psi(x, y)$

where $L_{x}$ and $L_{y}$ represents the lengths of our integration domain on the plane, and the kernel is the averged autocorrelation function $R\left(x, y ; x^{\prime}, y^{\prime}\right)=\left\langle u(x, y) u^{*}\left(x^{\prime}, y^{\prime}\right)>\right.$. The optimal basis elements $\Psi_{j}$ are provided by the eigenfunctions of this last equation, and are usually called "empirical eigenfunctions" because they can be obtained directly from experiments. The eigenvalues are a countable infinite set representing twice the average kinetic energy in each $\Psi_{j}$ mode, and they are ordered such that $\lambda_{j} \geq \lambda_{j+1}$. Finally from the diagonal decomposition of the autocorrelation function it follows that

$<a_{n}(t) a_{m}(t)>=\delta_{n m} \lambda_{n}$

that is, the temporal coefficients for different POD modes are uncorrelated on average and are related to the average kinetic energy. We could selectively chose a finite number $N$ of the most energetic modes and form a subspace spanned by the first $N$ eigenfunctions

$u_{N}(x, y, t)=\sum_{j=0}^{N} a_{j}(t) \Psi_{j}(x, y)$

which can be used as a reduced model of the dynamical behavior of the system we examined.

\section{Data analysis}

The line of sight velocity fields $u(x, y, t)$ are calculated from monochromatic images of the solar photosphere acquired on July 1, 1999 with the Italian Panoramic 

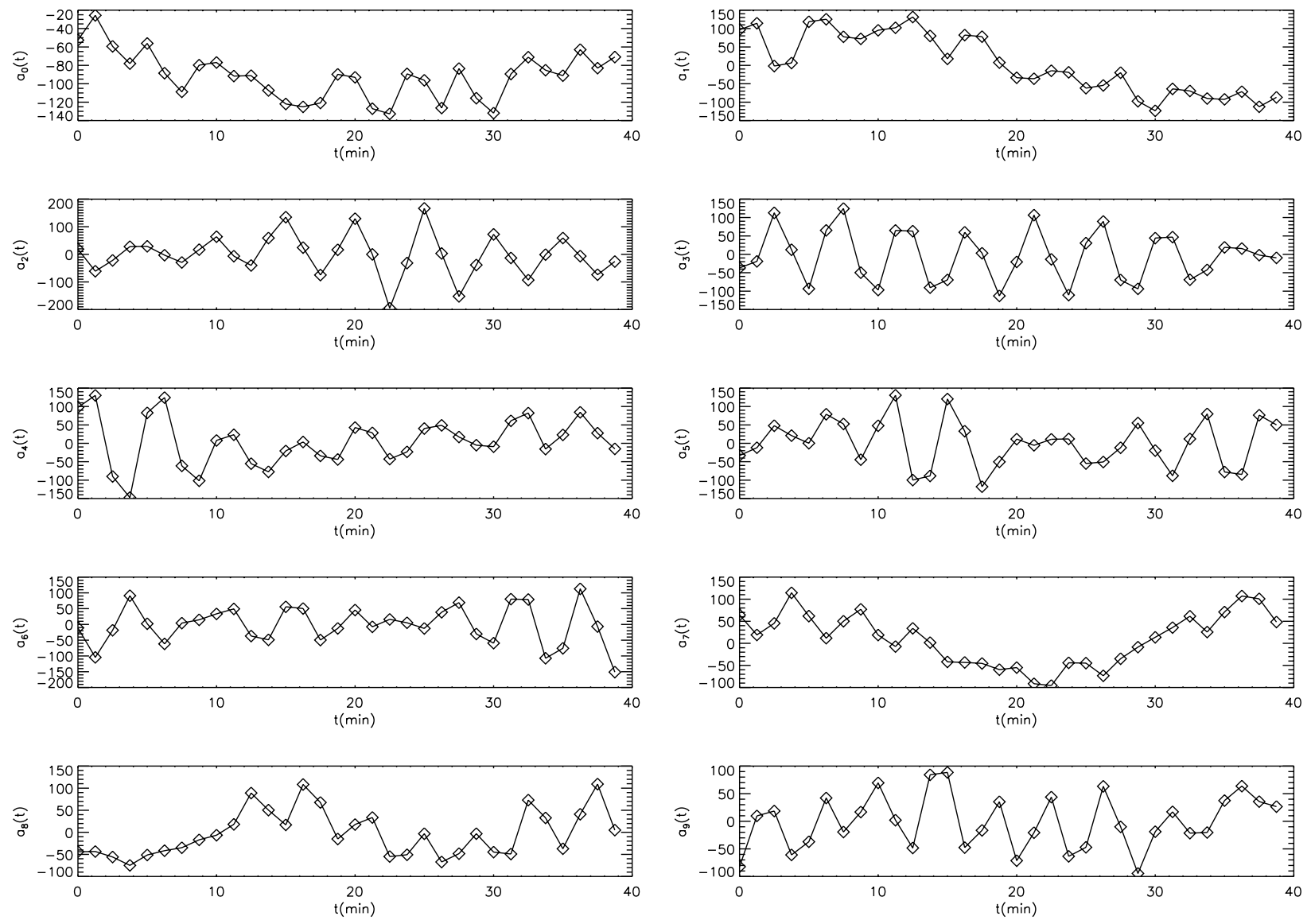

Fig. 2. Time evolution of the first ten coefficients $a_{j}(t)$ of the POD expansion.

Monochromator (Cavallini 1998) installed on the THEMIS telescope. The images refer to 7 spectral points within the Fe I $557.61 \mathrm{~nm}$ photospheric absorption line. This line is formed at a photospheric height of about $370 \mathrm{~km}$ (Komm et al. 1991) and the velocity fields are obtained from line Doppler-shifts. The size of the fields is $30^{\prime \prime} \times 30^{\prime \prime}$, the spatial resolution of the acquired images is about $0.4^{\prime \prime}$ while the resolution of the velocity fields is around $0.7^{\prime \prime}$. The analysed time series consist of 32 images which cover a time interval of about $40 \mathrm{~min}$. First we compared results from POD analysis with the results of the usual $k-\omega$ filter. Images were filtered by the standard technique, and the granular velocity field $u_{\mathrm{F}}(x, y, t)$ was obtained. Then, as a measure of the difference between POD analysis and $k-\omega$, we calculated the cross-correlation $\operatorname{Corr}\left(u_{N}, u_{\mathrm{F}}\right)$ of $u_{\mathrm{F}}(x, y, t)$ and $u_{N}(x, y, t)$. In Fig. 1 we report the time evolution of the maximum cross-correlation $\max _{N}\left\{\operatorname{Corr}\left(u_{N}, u_{\mathrm{F}}\right)\right\}$ obtained by allowing $N$ to be variable. Two modes suffice to reproduce the results obtained from the standard technique, the maximum correlation being about $70 \%$. This is true, except for the images which are at the boundaries of the temporal interval. This is probably due to spurious boundary effects in the $k-\omega$ analysis, when the Fourier transform is applied to non periodic functions like those from observations. In Fig. 2 we report the time evolution of the first ten coefficients $a_{j}(t)$ for $j=0,1, \ldots, 9$. It is evident that the $j=2,3$ modes correspond to the "5-min" oscillations. On the contrary, we can expect that the first two mode are related to the convective overshooting. This is confirmed by the spatial shape of the various modes which is represented by the eigenfunctions $\Psi_{j}(x, y)$. In Fig. 3 we report two cases corresponding to the $\Psi_{0}(x, y)$ mode (Fig. a), and the $\Psi_{2}(x, y)$ mode (Fig. b). As it can be seen, the first mode is characterised by the typical spatial scales of convective structures, while the second map, corresponding to oscillations, shows on average larger structures. This result agrees, roughly speaking, with the common finding that oscillations have predominantly smaller wavevectors than granules. In Fig. 4 we report the kinetic energy of each mode and the cumulative energy content of the modes $\left|\lambda_{j}\right|^{2}$ as a function of the mode number $j$. As it can be seen, the first four modes take more than $40 \%$ of the total energy. This indicates that, even if the $j=0,1$ modes dominate the energetics of overshooting motions, and the $j=2,3$ modes dominate the energetics of oscillations, subdominant contributions are important. These subdominant contributions are due to nonlinear coupling between the most energetic modes. These contributions cannot be filtered using the standard $k-\omega$ technique, because a spatial localization of modes is 

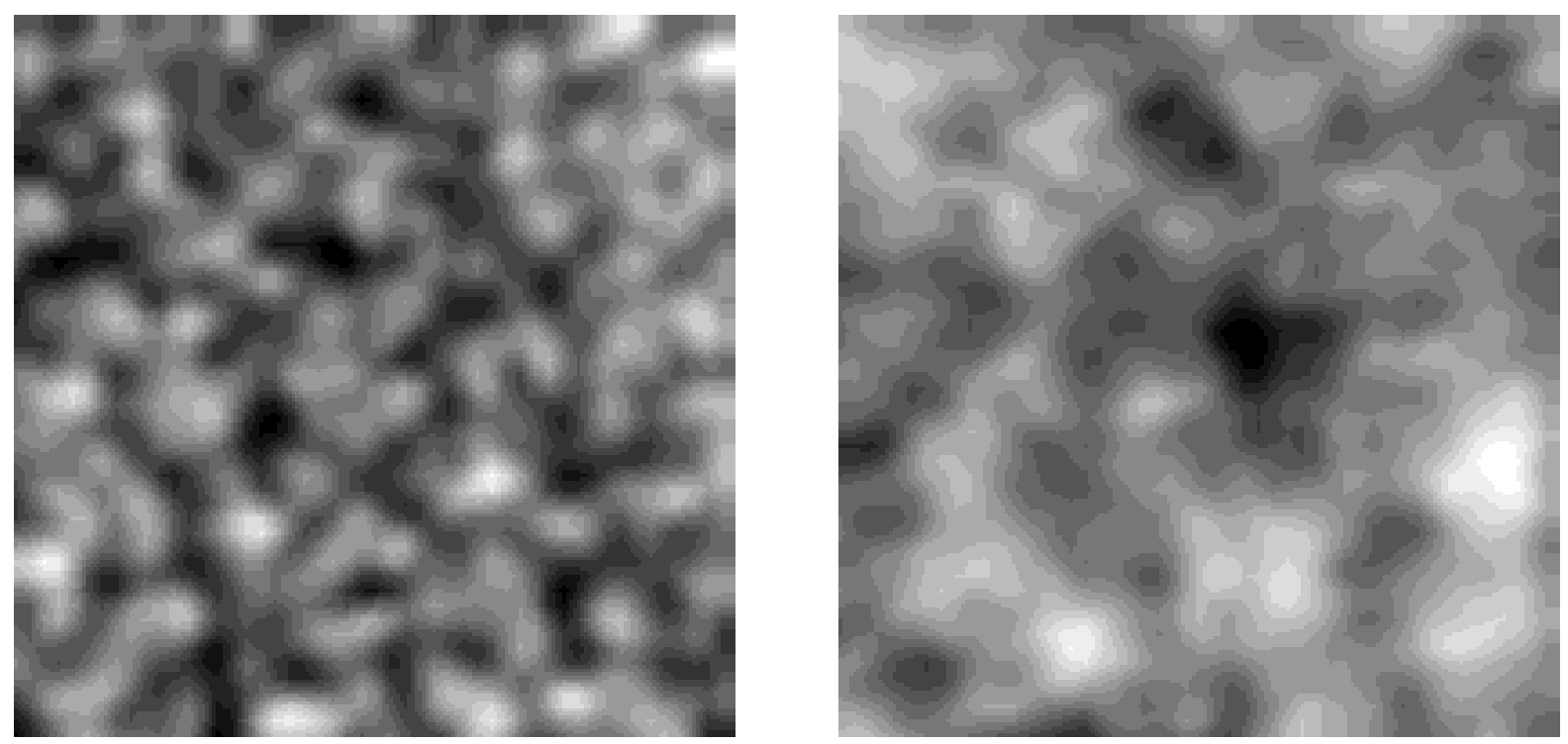

Fig. 3. In the left-hand panel we show the spatial pattern $\Psi_{0}(x, y)$, in the plane $(x, y)$, of the POD expansion. In the right-hand panel we show the spatial pattern $\Psi_{2}(x, y)$, in the plane $(x, y)$, related to $p$-modes.

not allowed by the Fourier analysis. Looking at the time evolution of the various modes, we can recognize contributions due to both convection and oscillations. In Fig. 5 we show an example of a velocity field calculated from Doppler shifts and the corresponding $k-\omega$ filtered field. In the same figure the velocity field

$u_{\mathrm{g}}(x, y, t)=\sum_{j=0,1} a_{j}(t) \Psi_{j}(x, y)$

reconstructed from the $j=0,1$ modes, and the velocity field

$u_{\mathrm{o}}(x, y, t)=\sum_{j=2,3} a_{j}(t) \Psi_{j}(x, y)$

reconstructed from the $j=2,3$ modes, are shown as a comparison.

\section{Discussion and conclusions}

In the present paper we presented a new application of POD to investigate both the $p$-modes and the convective pattern by using the longitudinal photospheric velocity field as measured by a solar telescope (in our case by THEMIS), the velocity fields at different times being used as an ensemble of different contributions to gain some insight into the complex spatio-temporal nature of photospheric motions. The POD is the most up-to-date technique used to capture the energetics of the phenomenon, giving information on the dynamics of the coherent structures, and providing an orthogonal empirical basis. In this respect the photospheric structures are identified as coherent structures which evolve stochastically in time. We now discuss the main results obtained.
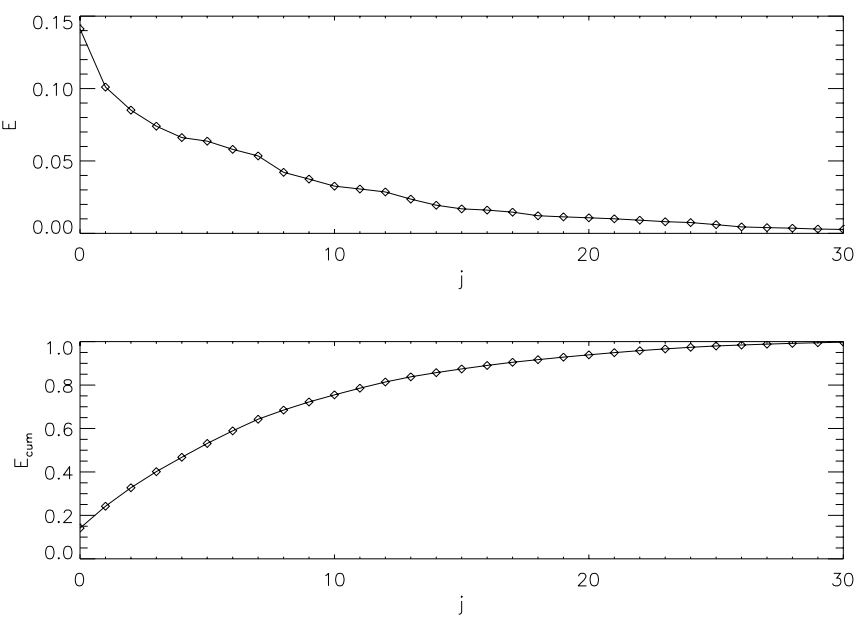

Fig. 4. The kinetic energy content of each eigenmode $j$ (upper panel), and the cumulative kinetic energy content of each eigenmode $j$ (lower panel), as a function of the mode number $j$.

1) Through the POD expansion we found that the first two modes $(j=0,1)$, where the energy is mainly concentrated, describe the pattern of convective structures, and the next two modes $(j=2,3)$ describe " 5 -min" oscillations. The pattern recovered from the first two modes is reasonably well correlated to the pattern obtained through the standard $k-\omega$ technique. However only $40 \%$ of the total energy is confined in the first four modes, the remaining fraction being spreaded among the next modes. Then, even if the first four modes suffice to reproduce both the convective pattern and the " $5-\min$ " oscillations, this is a strong indication that these most energetic modes interact to produce the modes at higher wave numbers. 
Original velocity field

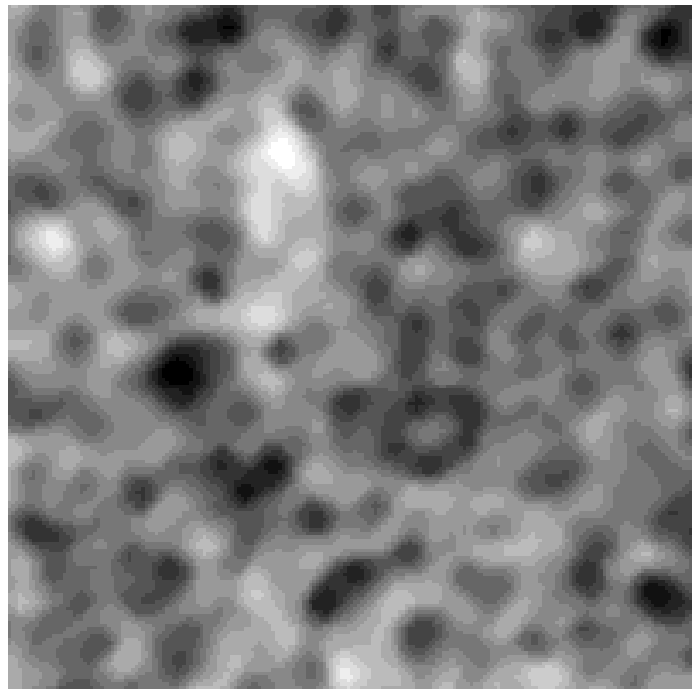

Reconstructed field with $j=0,1$ modes

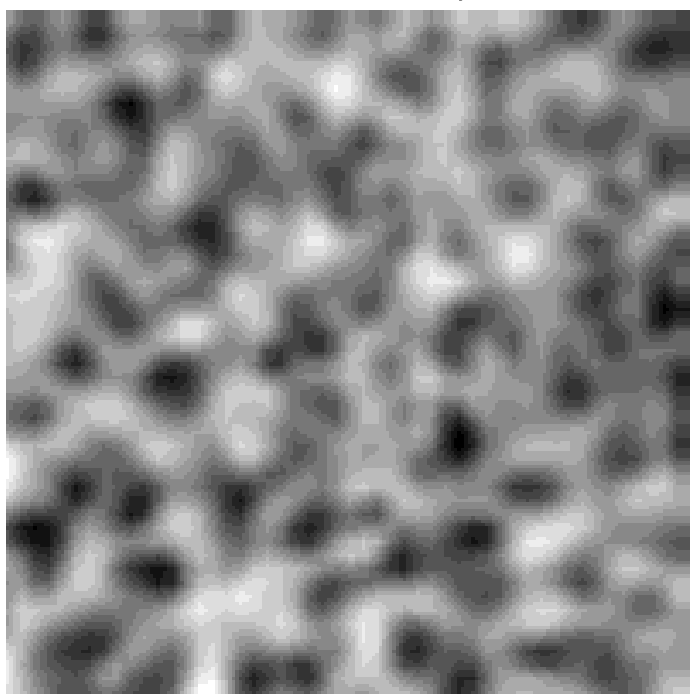

$k-\omega$ filtered velocity field

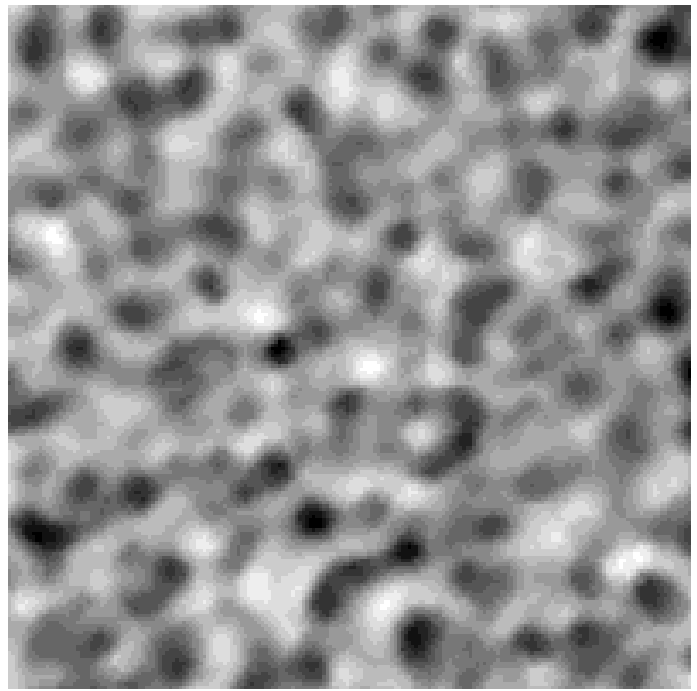

Reconstructed field with $j=2,3$ modes

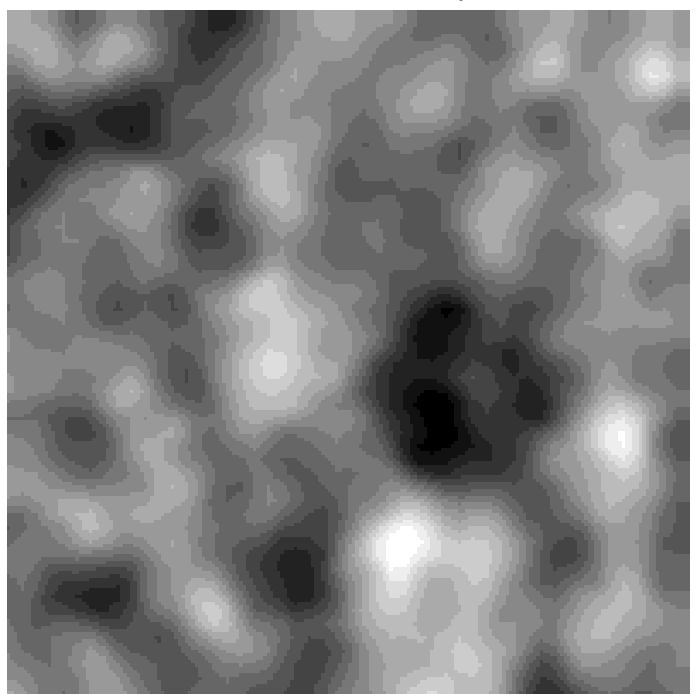

Fig. 5. An example line of sight velocity field calculated from Doppler shifts (upper left), the same velocity field filtered with the $k-\omega$ technique (upper right), and the velocity fields reconstructed from the $j=0,1$ modes (lower left) and from the $j=2,3$ modes (lower right) respectively.

2) We are able to reproduce the spatial pattern $\Psi_{j}(x, y)$ which contributes to the velocity field at each eigenmode. In other words, we can reconstruct not only the convective overshooting pattern, but also the spatial pattern associated with photospheric oscillations.

What we have found must be considered as early results of a technique which can be successfully applied to photospheric motions to gain insight into the physical behavior of convective and oscillatory motion, as well as their interactions. Indeed, little is known about the interaction between oscillations and convection in the solar atmosphere (Stix 1991). Convection might be a source of damping for oscillations, but it should be also seen as the main contribution to a stochastic excitation of the oscillatory behavior (Lighthill 1952; Goldreich \& Keeley 1977).
Our results can be used to improve knowledge in this area. Of course, we need longer time series, with a larger spatial extension, in order to better investigate the characteristic frequencies as well as spatial patterns of the $p$-modes, and in order to investigate the role played by meso-granular and/or super-granular cells. In the future we will build up a low-order model which describe the coupling between the $p$-modes and the granulation. This can be made using the empirical eigenfunctions to make a Galerkin approximation of equations describing the phenomenon. A future paper will be devoted to this interesting topic.

Acknowledgements. We thank the THEMIS staff for efficient support in the observations. This work was partially supported by the Italian National Research Council (CNR) grant Agenzia2000 CNRC0084C4. 


\section{References}

Bray, R. J., Loughhead, R. E., \& Durrant, C. J. 1984, The Solar Granulation, 2nd ed. (Cambridge University Press, Cambridge)

Cavallini, F. 1998, A\&A, 128, 589

Consolini, G., Carbone, V., Berrilli, F., et al. 1999, A\&A, 344, L33

Goldreich, P., \& Keeley, D. A. 1977, ApJ, 212, 243

Holmes, P., Lumley, J. L., \& Berkooz, G. 1998, Turbulence, coherent structures, dynamical systems and symmetry (Cambridge University Press, Cambridge)
Komm, R., Mattig, W., \& Nesis, A. 1991, A\&A, 252, 812

Leighton, R. B., Noyes, R. W., \& Simon, G. W., 1962, ApJ, 135,474

Lighthill, M. J. 1952, Proc. R. Soc. London A, 211, 564

Lumley, J. L. 1967, in Atmospheric turbulence and wave propagation, ed. A. M. Yaglom, \& V. I. Tatarski (Nauka, Moscow), 166

Stix, M. 1991, The Sun: An introduction (Springer Verlag, Berlin)

Straus, Th., Deubner, F. L., \& Fleck, B. 1992, A\&A, 256, 652

Title, A. M., Tarbell, T. D., Topka, K. P., et al. 1989, ApJ, 336, 475 